\title{
Molecular pathology of prostate cancer: a practical approach
}

\author{
Tatjana Vlajnic, Lukas Bubendorf \\ Institute of Pathology, University Hospital Basel, Basel, Switzerland
}

\begin{abstract}
Summary
While localised prostate cancer can be cured by local treatment, 'high-risk' prostate cancer often progresses to castration resistant disease and remains incurable with a dismal prognosis. In recent years, technical advances and development of novel methodologies have largely contributed to a better understanding of underlying molecular mechanisms that promote tumour growth and progression. Consecutively, novel therapeutic strategies for treatment of prostate cancer have emerged during the last decade, calling for the identification of predictive biomarkers. The concept of personalised medicine is to tailor treatment according to the specific tumour profile of an individual patient. Moreover, acquired molecular changes during tumour evolution and in response to therapy selection pressure require adapted predictive marker testing at different time points during the disease. In this setting, the pathologist plays a critical role in patient management and treatment selection.

In this review, we provide a comprehensive overview of the current knowledge of molecular aspects of prostate cancer and their potential utility in the context of different therapeutic approaches. Furthermore, we discuss methods for molecular marker testing in routine clinical practice, with a focus on castration resistant prostate cancer.
\end{abstract}

Key words: Prostate cancer; molecular biomarkers; prognostic markers; predictive markers; CRPC; AR-V7; HRD; DNA repair deficiency.

Received 11 October, accepted 14 October 2020

Available online 21 November 2020

\section{INTRODUCTION}

Prostate cancer is a clinically and biologically heterogeneous disease, which requires different treatment strategies depending on the clinical setting. In patients with newly diagnosed localised prostate cancer, decisions for radical local treatment or active surveillance largely depend on tumour-related factors such as tumour extent on biopsies, histological subtype, Gleason/International Society of Urological Pathology (ISUP) grade, and serum prostate specific antigen (PSA) level. However, in order to prevent overtreatment, there is a need for additional prognostic markers that can more accurately predict biological behaviour of the tumour and support clinical decision making. Apart from the above mentioned factors, Gleason/ISUP grading being the most powerful predictor of clinical outcome, particularly PTEN in combination with ERG, and the proliferation index
$\mathrm{Ki}-67$, are emerging as markers with additional prognostic value.

On the other hand, patients who initially present with locally advanced or metastatic prostate cancer show variable duration of the disease and usually suffer several relapses in the castration sensitive and eventually castration resistant stage. As androgen receptor (AR) signalling pathway plays a major role in prostate cancer development and progression, systemic treatment is primarily based on antiandrogen therapy (androgen deprivation therapy, ADT), optionally combined with taxane-based chemotherapy. Through technical progress and development of novel methodologies, we have gained new insights into underlying molecular mechanisms that promote tumour growth and castration resistance. Identification of novel potential therapeutic targets involving the recurrently altered DNA repair pathway and phosphoinositide 3-kinase (PI3K)-AKT-mTOR signalling pathway has expanded the treatment landscape in castration resistant prostate cancer (CRPC). Notably, the recognition of a reactivation of the AR signalling pathway in CRPC has led to the development of novel potent AR-targeted drugs such as the second-generation antiandrogens abiraterone and enzalutamide. However, there is still lack of consensus for the optimal treatment sequence and use of combinational treatment strategies. ${ }^{1}$ Therefore, there are ongoing efforts to establish predictive biomarker testing for various treatment options and identify possible primary and secondary resistance mechanisms. By tailoring biomarker testing at different time points during the disease depending on the clinical setting, pathologists play a key part in the management of prostate cancer patients and contribute to treatment optimisation.

Here, we give an overview of the currently available biomarkers that can be useful to guide treatment decisions in clinical practice, with a focus on CRPC.

\section{BIOMARKERS IN LOCALISED PROSTATE CANCER}

PTEN in combination with ERG

The tumour suppressor gene PTEN is one of the most commonly altered genes in prostate cancer. Inactivation of PTEN occurs in $10-20 \%$ of organ-confined PC, ${ }^{2}$ whereas it has been identified in up to $40 \%$ of metastatic PC. ${ }^{3}$ PTEN loss results in activation of the PI $3 \mathrm{~K}-\mathrm{AKT}-\mathrm{mTOR}$ signalling pathway with subsequent increase of cell growth and proliferation. Hence, PTEN loss is strongly associated with adverse pathological factors as well as clinical outcome (summarised by Jamaspishvili et $a$. $^{3}$ ) and therefore appears suitable as a prognostic biomarker. Especially in the setting 
of low volume and low Gleason grade prostate cancer, PTEN could help stratifying patients to active surveillance or definitive local treatment. Traditionally, a FISH assay has been used to detect PTEN loss, which is most often caused by a homozygous deletion. Rarely, PTEN can be silenced by an inactivating mutation, a genetic rearrangement or by epigenetic mechanisms. ${ }^{3,4}$ Meanwhile, there is a validated and robust immunohistochemistry (IHC) assay for detection of PTEN protein loss as a surrogate marker for PTEN inactivation regardless of the underlying mechanism. ${ }^{5}$

Only complete absence of PTEN staining or extremely weak intensity staining in $>10 \%$ of tumour cells should be considered negative for PTEN (Fig. 1). ${ }^{5}$ Although weak expression could reflect a heterozygous PTEN deletion in some cases, the interpretation of varying expression intensities by IHC is unreliable and not recommended. Intratumoural heterogeneity of PTEN loss (defined as PTEN loss in $>10 \%$ and $<100 \%$ of the tumour cells) occurs in up to $40 \%$ of cases. Its prognostic relevance has been less well studied but the prognosis seems to be intermediate between homogeneous PTEN loss and intact PTEN. ${ }^{3,6}$

Although PTEN loss is regarded as an early event in tumourigenesis, enrichment of PTEN loss in ERG-rearranged prostate cancer supports the concept that it occurs subsequent to $E R G$ gene rearrangement. ${ }^{3,7-12}$ ERG expression as a result of TMPRSS2-ERG fusion is found in approximately $50 \%$ of organ-confined PC. ${ }^{13}$ ERG by itself has no proven prognostic value in surgically treated prostate cancer. ${ }^{14}$ Intriguingly, it has been shown that PTEN loss predicts the worst clinical outcome in the absence of $E R G$ rearrangement as compared to PTEN loss in ERG-positive prostate cancer. ${ }^{15,16}$ These findings emphasise that combined PTEN and ERG assessment can more accurately stratify prostate cancer risk of progression than PTEN analysis alone (Fig. 2).

Beside the prognostic value of PTEN loss, emerging clinical trial data suggest a potential role of PTEN as a predictive marker for response to PI3K inhibitors in the metastatic setting. ${ }^{17}$ Given the cost effectiveness and reliability of the IHC assay, systematic PTEN IHC analysis could easily be implemented in biopsies from patients with metastatic prostate cancer.

\section{Proliferation index Ki-67}

$\mathrm{Ki}-67$ labelling index (Ki-67 LI) is a well-established marker for proliferating cells and is routinely used to estimate tumour

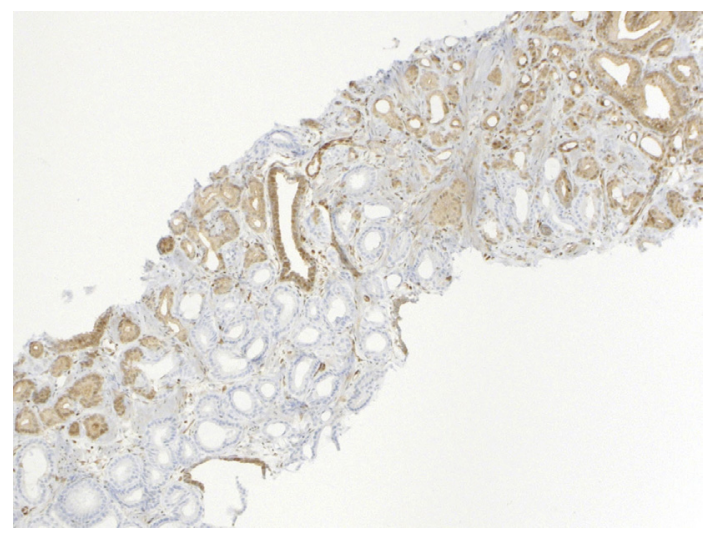

Fig. 1 IHC showing heterogeneous PTEN loss of expression in a Gleason score 6 prostate carcinoma: complete absence of staining in a subset of tumour cells (clone 138G6, 1:50 diluted, Cell Signaling; Ventana Benchmark Ultra). aggressiveness across different tumour types. ${ }^{18-23}$ In prostate cancer, an independent prognostic role in localised tumours has been shown in various studies as recently summarised in a meta-analysis. ${ }^{24}$ In this setting, Ki-67 IHC testing could help in stratifying patients for AS or local treatment. Although assessment of Ki-67 LI by IHC is not technically challenging, it is still not widely used in routine work-up of prostate carcinoma cases. $^{25}$ A major hurdle in interpretation of Ki-67 LI is the lack of consensus for an optimal threshold for high and low proliferation. Difficulties to determine a cutoff value, partly due to different scoring methods and interobserver variability, have already been addressed in other tumour types such as breast cancer. ${ }^{26}$ For the present time, there is general agreement that cut-off values for high and low proliferation should be defined for each institution individually. $^{27-29}$ In our experience, the proliferation index varies between 5 and $15 \%$ in the majority of prostate carcinomas and hardly ever exceeds $25 \%$. Given this rather small range, the cut-off for high and low proliferation would be around $10 \%$. Several other issues regarding $\mathrm{Ki}-67$ scoring remain to be clarified and standardised, including tumour heterogeneity in needle biopsy samples, evaluation of hot spots versus whole area, and the method of scoring (conventional analysis by a pathologist versus automated image analysis). Finally, before Ki-67 testing is routinely implemented in clinical practice, further studies are urgently needed to prospectively validate its role in the setting of curative treatment. While stratification around a $10 \%$ threshold remains a challenge for the time being, calling cases with very low (e.g., $<5 \%$ ) and clearly high Ki-67 LI (e.g., >15\%) is straightforward in our experience (Fig. 3). Overall, the combined analysis of Ki-67 LI, PTEN and ERG in biopsies has promise to provide useful prognostic information to guide management of individual patients with prostate cancer. However, further studies are needed before these analyses can be recommended for routine practice. ${ }^{25,30}$

\section{RNA-based genomic signatures}

In recent years, several RNA-based commercialised molecular expression assays have been proposed for assessment of prognosis and tumour aggressiveness, including Prolaris (Myriad Genetics, USA), Oncotype DX (Genomic Health, USA), and Decipher (GenomeDX Biosciences, USA). Their advantage of centralised and standardised testing with objective results needs to be weighed against the high costs and concerns raised about sampling bias in needle biopsy specimens. ${ }^{31}$ In the United States, Prostate Cancer National Comprehensive Cancer Network (NCCN) guidelines (version 2.2020) include molecular testing as a consideration in patients with low or favourable intermediate-risk disease if life expectancy is $\geq 10$ years. However, their utility remains controversial, and routine use is not recommended by the American Society of Oncology. ${ }^{30}$ In Europe, they are not yet widely used mostly due to lack of a prospective validation and lack of comparison with more economical IHC assays. ${ }^{25}$

\section{BIOMARKERS IN ADVANCED PROSTATE CANCER}

\section{Androgen receptor signalling pathway}

Advanced prostate cancer treated with ADT inevitably progresses to castration resistant disease. Different molecular 


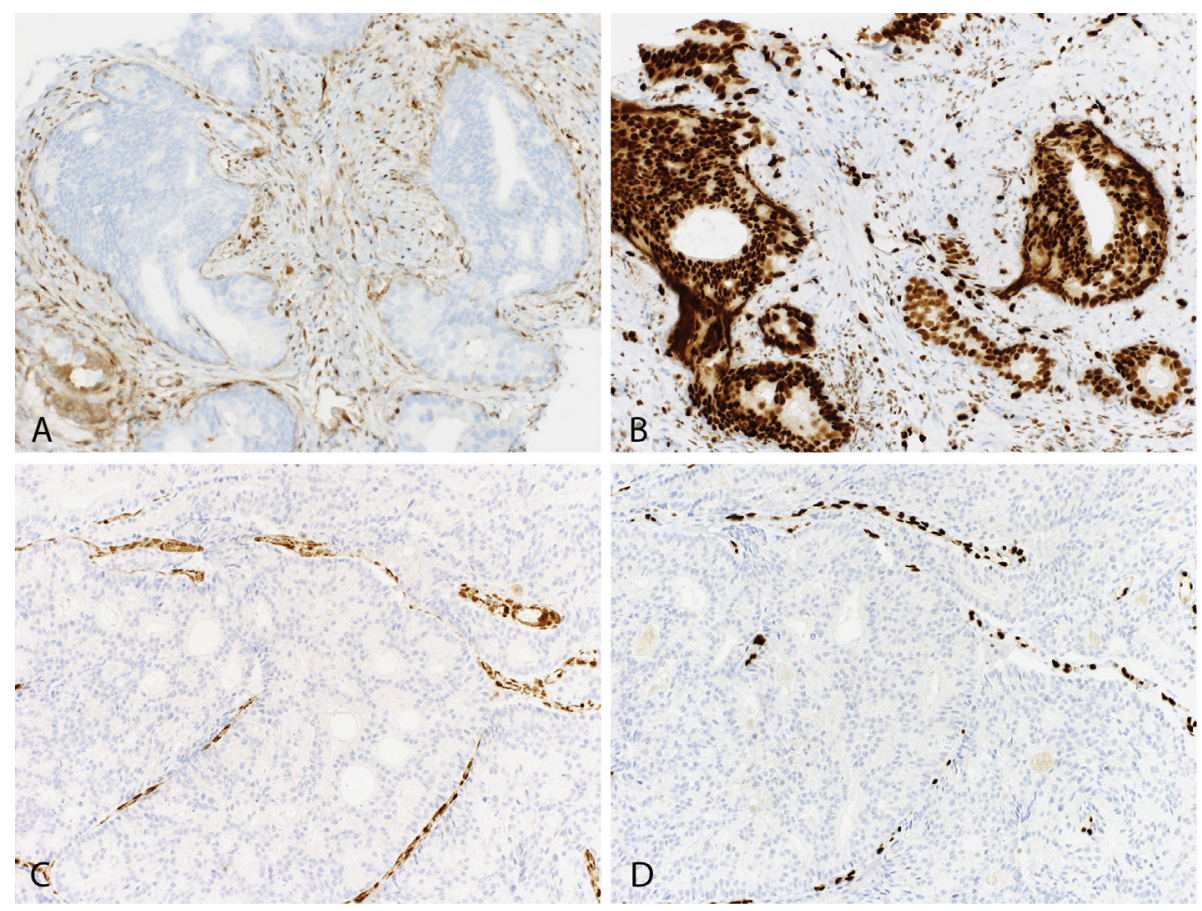

Fig. 2 Different PTEN and ERG IHC status in two cases with cribriform Gleason pattern 4 prostate carcinomas. (A) Complete loss of PTEN expression and (B) concomitant ERG positivity. (C) Complete loss of PTEN expression in (D) an ERG negative tumour. Note endothelial cells as internal positive control for both PTEN and ERG IHC (ERG: clone EPR3864, prediluted, Ventana; Ventana Benchmark Ultra).
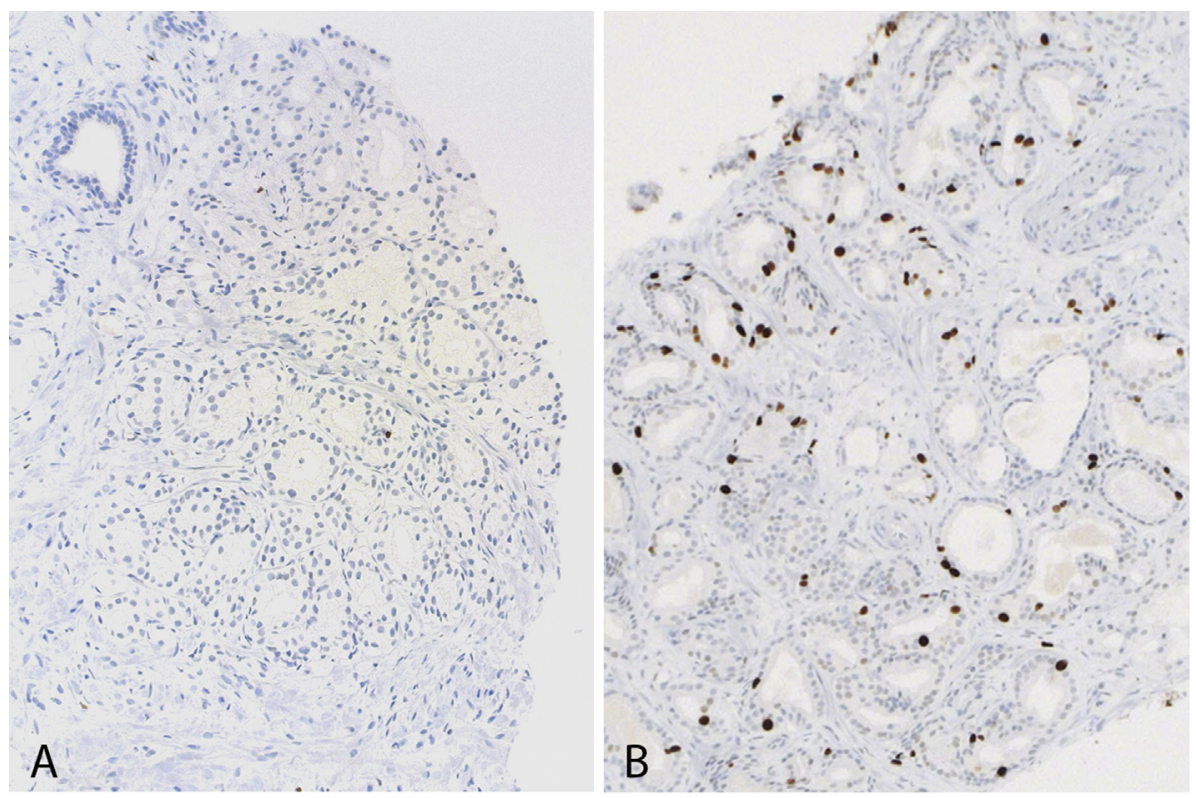

Fig. 3 Variabilities in proliferation index (Ki-67 LI) in two different cases of Gleason score 6 prostate carcinoma. (A) Low Ki-67 LI (<5\%) with IHC staining in scattered tumour cells. (B) High Ki-67 of approximately 15-20\% (clone MIB1, prediluted, Dako; Ventana Benchmark Ultra).

mechanisms have been identified that drive castration resistance, most importantly the reactivation of the AR signalling pathway. ${ }^{32}$ This mainly occurs through amplifications or mutations of the $A R$ gene as well as expression of AR splice variants, most commonly AR splice variant 7 (AR-V7). In a landmark study, Antonarakis et al. ${ }^{33}$ showed that AR-V7 mRNA expression in circulating tumour cells (CTCs) may predict poor response to the AR signalling inhibitors enzalutamide and abiraterone. Although this finding was not consistently reproducible in subsequent studies, ${ }^{34}$ Armstrong et al. showed that detection of AR-V7 in CTCs was independently associated with adverse outcome in patients treated with enzalutamide or abiraterone. ${ }^{35}$ Analysis of CTCs remains technically challenging and therefore is not yet widely implemented. We have validated a commercially available test for analysis of AR-V7 on CTCs that can be used to determine the AR-V7 status in patients with CRPC. ${ }^{36}$ In addition, antibodies have become available allowing to better analyse AR-V7 expression in formalin-fixed paraffinembedded (FFPE) tissue ${ }^{37}$ (Fig. 4). Predominantly nuclear AR-V7 expression has seldom $(<1 \%)$ been found in hormone-naïve prostate cancer but in $75 \%$ of CRPC, where it was associated with adverse outcome under endocrine therapies. ${ }^{37}$ Therefore, AR-V7 status should ideally be studied at 


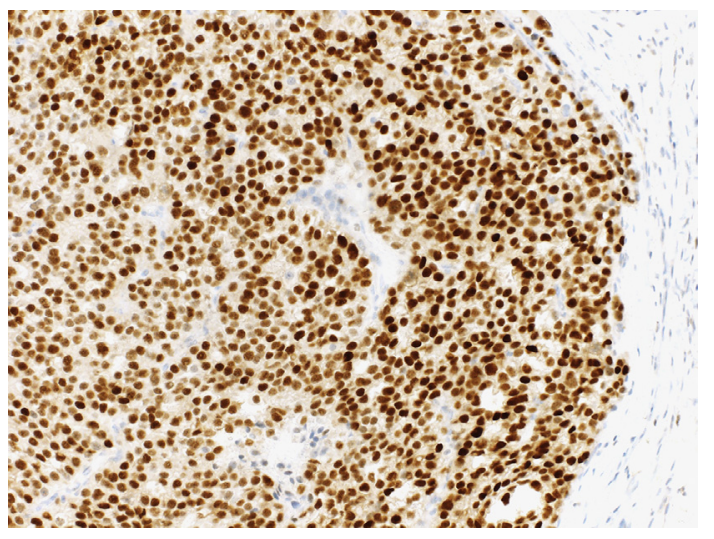

Fig. 4 IHC showing diffuse nuclear expression of AR-V7 in castration resistant prostate cancer (clone RM7, 1:25 diluted, Dianova; Ventana Benchmark Ultra).

the time of recurrence after ADT and not in earlier pretreatment specimens. Also $A R$ gene alterations can be assessed on tissue biopsies of CRPC. Both hot spot $A R$ mutations and $A R$ amplifications can be detected by nextgeneration sequencing (NGS). Alternatively, fluorescence in situ hybridisation can be used as the gold standard to quantify $A R$ gene copy number. $A R$ amplification occurs in up to $40 \%$ of CRPC but almost never in untreated prostate cancer $^{38}$ (Fig. 5). Although the predictive role of $A R$ amplification remains to be explored, it has been identified as an adverse prognostic marker in cell-free DNA (cfDNA) from patients with metastatic CRPC (mCRPC). ${ }^{39-41}$

\section{Neuroendocrine differentiation in prostate cancer}

Testing for neuroendocrine markers has gained increasing attention in the setting of castration resistance, since neuroendocrine differentiation is one of the resistance mechanisms to antiandrogen therapy. ${ }^{42}$ These patients more often present with rapid progression and visceral metastases despite relatively low PSA values. Diagnosis of small cell neuroendocrine carcinoma requires both expression of at least one neuroendocrine marker (synaptophysin, chromogranin, or CD56) and the specific morphology. Since small cell carcinoma emerges in nearly one-fifth of patients with CRPC, the diagnosis requires a contemporary biopsy but not a reanalysis of the initial biopsy prior to treatment. ${ }^{42}$ A primary

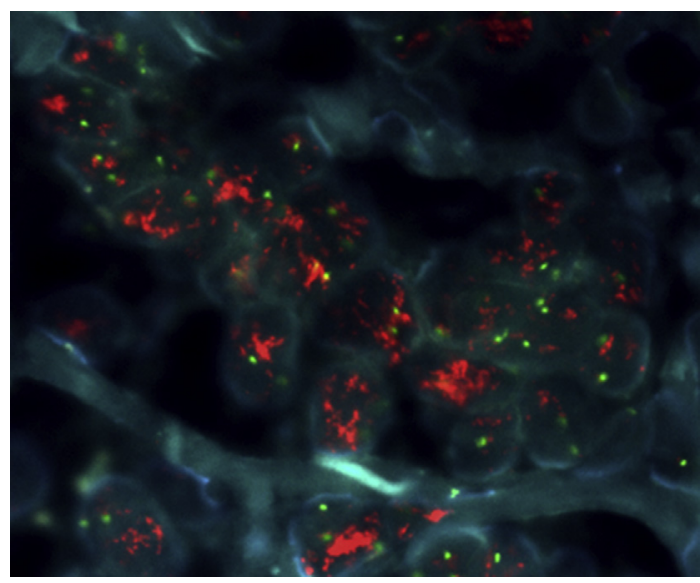

Fig. 5 FISH showing a high-level amplification of the $A R$ gene (clusters of red signals) and normal copy number of CEP X reference signals (green signals) in castration resistant prostate cancer. small cell carcinoma of the prostate at initial diagnosis is rare. ${ }^{43}$ In cases of conventional morphology acinar or ductal prostate carcinoma, especially at initial diagnosis, routine immunohistochemical testing with neuroendocrine markers is not specific and therefore not recommended. ${ }^{25}$ Scattered cells with neuroendocrine differentiation, referred to as focal neuroendocrine differentiation, are often present in an otherwise conventional prostate carcinoma and have no diagnostic or prognostic implications. ${ }^{44}$ Some cases of newly diagnosed conventional prostate carcinoma, however, especially with high Gleason pattern, may exhibit a diffuse expression of neuroendocrine markers. This phenomenon is not yet well studied but seems to have limited prognostic value. $^{45,46}$ CRPC with neuroendocrine differentiation harbour mutations in RB1 and TP53 genes in up to $70 \%$ of cases each, and concurrent mutations in approximately $50 \%$. However, these mutations are not specific for neuroendocrine differentiation. $^{47}$

Immunohistochemical testing for TTF1 is not helpful for excluding metastatic small cell carcinoma of the lung as TTF1 is often expressed in small cell carcinomas irrespective of their primary site. The only marker specific for prostate origin is TMPRSS2-ERG rearrangement. Whereas ERG expression is usually lost after transdifferentiation to small cell carcinoma due to inactivated AR signalling, the rearrangement remains detectable by FISH or RNA-based NGS methods. ${ }^{48}$

\section{DNA repair deficiency}

\section{Homologous recombination DNA repair deficiency}

In recent years, major research activity has focused on DNA repair deficiency, leading to development of novel therapeutic approaches such as poly ADP ribose polymerase (PARP) inhibitors. ${ }^{49,50}$ PARP inhibitors show therapeutic effects in tumours with deficiency in the homologous recombination DNA repair pathway (homologous recombination deficiency, HRD), which can be caused by either genetic or epigenetic mechanisms. More specifically, current hypothesis indicates that PARP inhibitors are effective only in case of loss of function or biallelic inactivation of HRD related genes (i.e., germline mutation plus somatic loss, or double-somatic alterations). ${ }^{51}$ Impressive responses to the PARP inhibitor olaparib were observed in patients with advanced ovarian and breast carcinomas harbouring germline mutations in BRCA1 and BRCA2 genes. ${ }^{52,53}$ In addition, some $B R C A$-wildtype ovarian carcinomas equally seem susceptible to PARP inhibitors, ${ }^{54}$ emphasising the need for biomarkers of HRD signature ('BRCAness' phenotype). In multicentre sequencing studies of advanced prostate cancer, mutations in HRD associated genes, most commonly in $B R C A 2$, were identified in approximately $20-25 \%$ of mCRPC. ${ }^{55,56}$ Notably, half of these patients had germline mutations in these genes, which is significantly higher compared to the incidence in patients with localised prostate cancer. ${ }^{57}$ Presence of HRD is associated with aggressive histological features including Gleason pattern 5 and intraductal/ductal prostate carcinoma. ${ }^{58-62}$ In one of the first trials, olaparib demonstrated greatest efficacy in mCRPC patients harbouring genetic alterations in genes directly or indirectly involved in HRD. ${ }^{63}$ Recently published data from the phase 3 PROfound trial provide further evidence for olaparib activity in $\mathrm{MCRPC}$ with HRD, especially in patients with $B R C A 1, B R C A 2$, or ATM alterations. ${ }^{64}$ Ongoing trials 
are evaluating other PARP inhibitors including niraparib and rucaparib $^{65-67}$ as well as combination approaches with immune checkpoint inhibitors. ${ }^{68}$ Moreover, emerging evidence suggests that, similarly to ovarian and breast carcinoma, patients with HRD-associated mCRPC may also benefit from platinum-based chemotherapy. ${ }^{69-71}$ Consequently, molecular stratification of mCRPC with a focus of HRD screening is increasingly becoming standard in clinical practice. Overall, it seems that greatest benefit from both PARP inhibitors and platinum-based chemotherapy occurs in $B R C A 1$-and BRCA2-associated tumours as compared to tumours with any of the other HRD-related genes. NGS is the most widely used method for mutational testing and should at a minimum include the $B R C A 1$ and $B R C A 2$ genes. Ideally, testing should cover other HRD-related genes (ATM, CHEK2, PALB2, FANCA, RAD51D, and CDK12), as currently recommended by the NCCN guidelines. ${ }^{72}$ The optimum time point for molecular analyses yet remains to be defined. According to expert opinion, molecular testing on a fresh biopsy of a metastatic lesion would be optimal. ${ }^{73}$ Given that most HRD-related gene mutations occur before castration resistance ${ }^{74}$ and fresh tumour tissue is not always available, HRD analysis can be performed on archival FFPE material from the primary tumour biopsy or prostatectomy specimen. Alternative analysis of CTCs or circulating tumour DNA (ctDNA) is technically more challenging and currently under validation. ${ }^{75}$ When there is a lack of any tumour tissue, germline testing remains an option. However, germline testing misses the other half of somatic HRD-related gene mutations in patients with $\mathrm{mCRPC}$.

In a subset of tumours, NGS testing may reveal mutations of unknown significance (so called variants of unknown significance, VUS) in HRD-related genes. In this scenario, there is the possibility to determine the HRD score, as a surrogate marker, to estimate the probability of a pathogenic mutation. ${ }^{76}$ The HRD score is a measure of chromosomal aberrations that are related to HRD.

Preliminary data suggest that biallelic inactivation of the CDK12 gene induces an immunogenic state, providing a target for immune checkpoint inhibitor (ICI) therapy. ${ }^{77}$

\section{Mismatch repair deficiency and microsatellite instability}

Another mechanism of DNA repair frequently altered in tumours is the mismatch repair (MMR) pathway. Loss of function of one of the four MMR proteins MLH1, MSH2, MSH6, and PMS2, results in a defective MMR pathway (dMMR) and microsatellite instability (MSI). Defective MMR causes a hypermutated tumour phenotype resulting in genomic instability and increased neoantigen burden. Overall, defects in MMR pathway are rare in prostate cancer, but they are enriched in CRPC, occurring in up to $10 \%$ of CRPC. ${ }^{78-80}$ Striking responses to ICI have been observed in tumours with MSI-high or dMMR, subsequently leading to a tumour-agnostic approval for ICI treatment in tumours harbouring these alterations. ${ }^{81,82}$ Moreover, prostate cancers with dMMR are sensitive to hormonal therapies. ${ }^{83}$ MMR status can reliably be determined using a routine IHC panel with the antibodies against MLH1, MSH2, MSH6, and PMS2. In prostate cancer, dMMR most commonly occurs due to loss of expression of MSH2, variably associated with MSH6 loss of expression. ${ }^{84}$ Importantly, loss of expression can be heterogeneous ${ }^{85}$ but the impact of this heterogeneity on response to ICI has not yet been investigated (Fig. 6).

\section{Prostate-specific membrane antigen}

Overexpression of prostate-specific membrane antigen (PSMA) has been associated with high-grade and advanced
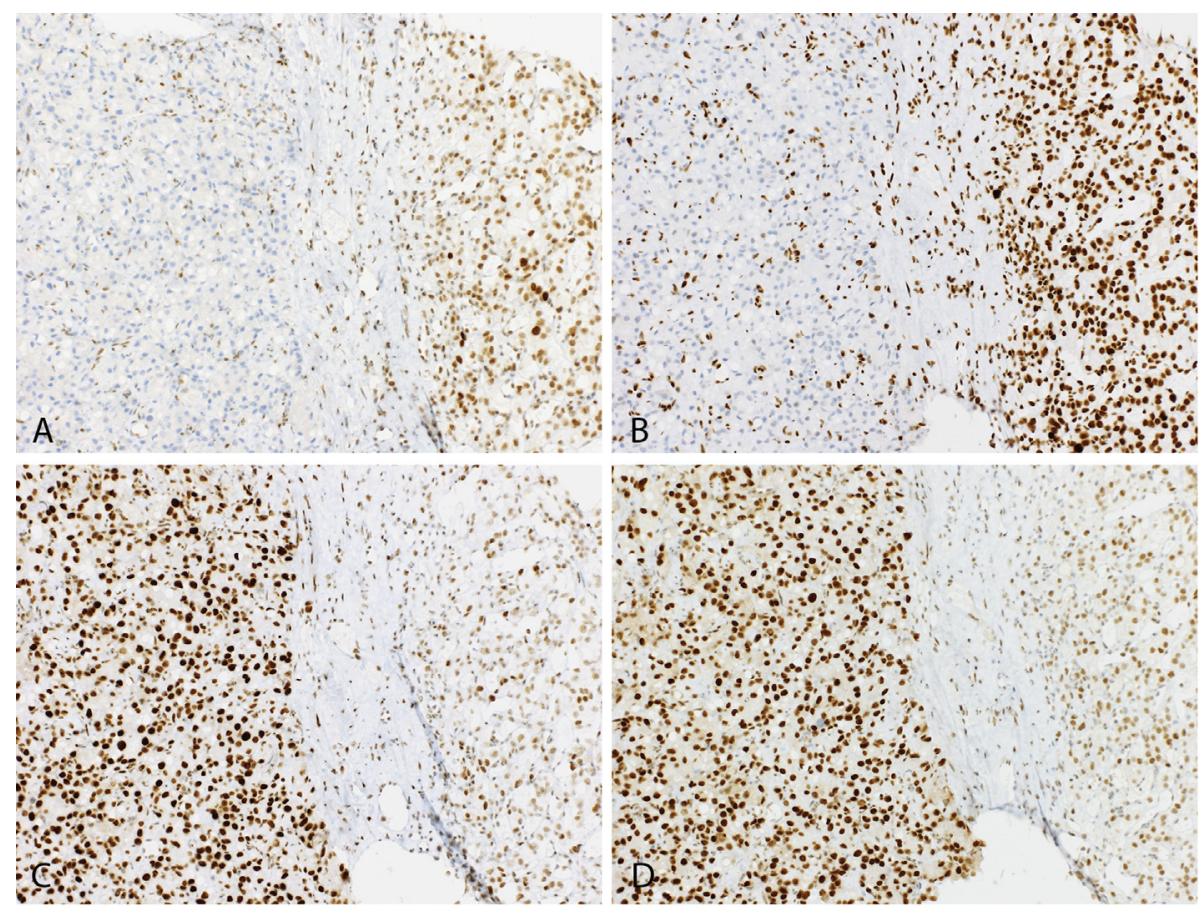

Fig. 6 IHC showing microsatellite instability in a treatment naïve prostate cancer biopsy. (A,B) Heterogeneous loss of expression of MSH2 and MSH6, respectively, in one part of the tumour (left side; note preserved internal positive control in endothelial cells) and intact expression in the other part (right side). (C,D) Preserved expression of MLH1 and PMS2 in the tumour (MSH2: clone G219-1129, prediluted, Ventana; MSH6: clone SP93, prediluted, Ventana; MLH1: clone M1, prediluted, Ventana; PMS2: clone EP51, prediluted, Dako; Ventana Benchmark Ultra). 

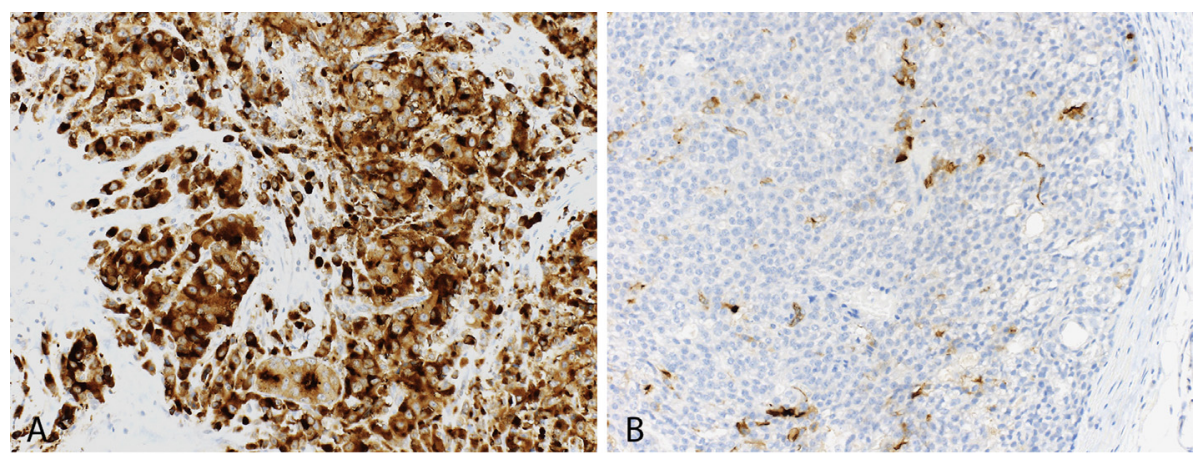

Fig. 7 IHC showing variable PSMA expression in two different castration resistant prostate cancer cases. (A) Diffuse cytoplasmic PSMA expression and (B) focal PSMA expression in scattered tumour cells (clone EP192, prediluted, Ventana; Ventana Benchmark Ultra).

stage prostate cancers as well as higher risk for biochemical recurrence, suggesting a role as prognostic marker (Fig. 7). ${ }^{86,87}$ Only recently, PSMA-targeted therapies labelled with beta emitters like Lutetium-177 $\left({ }^{177} \mathrm{Lu}\right)$ for radioligand therapy have emerged as a promising therapeutic option for patients with mCRPC. ${ }^{88,89}$ Patients are screened for eligibility according to high PSMA uptake in the metastases as detected by ${ }^{68} \mathrm{Ga}$-labelled PSMA-positron emission tomography $\left({ }^{68} \mathrm{Ga}-\mathrm{PSMA}-\mathrm{PET}\right)$. Likewise, ${ }^{68} \mathrm{Ga}-\mathrm{PSMA}-\mathrm{PET}$ is used to define response to ${ }^{177} \mathrm{Lu}$-PSMA treatment. Preliminary studies showed a low proportion of tumour staining $(<50 \%)$ by IHC was strongly associated with a negative ${ }^{68} \mathrm{Ga}-$ PSMA-PET scan. ${ }^{90,91}$ However, the role of PSMA expression by IHC as a predictive marker in the setting of PSMAtargeting therapies is yet to be defined. The value of tissuebased PSMA testing to tailor and monitor PSMA-targeted treatment remains to be analysed in future studies.

\section{Cell-free DNA analysis}

The emergence of established or potential predictive genomic markers in metastatic and castration resistant prostate cancer has led to increasing demands for genomic testing. ${ }^{92}$ This development is currently mainly driven by the need to test for mutations of HRD genes including BRCA1, BRCA2, and $A T M{ }^{93}$ Since re-biopsies for genomic testing are often difficult to obtain in patients with CRPC, plasma cell-free DNA (cfDNA) appears as a practical, minimally-invasive solution to identify predictive or prognostic genomic alterations, and to monitor therapy response. ${ }^{92}$ In fact, previous sequencing studies showed a high overlap between contemporary biopsies and cfDNA. ${ }^{94}$ Continuous methodological advances in sequencing technology have further increased the accuracy of cfDNA analysis. ${ }^{95}$ Although there are commercial pan-cancer cfDNA assays for centralised panel NGS analysis including $B R C A 1$ and $B R C A 2$, e.g., FoundationOne Liquid CDx (Foundation Medicine, USA) ${ }^{96}$ or Guardant 360 (Guardant Health, USA), ${ }^{97,98}$ prostate-specific panels for decentralised testing are in development but not yet commercially available. ${ }^{92}$

\section{CONCLUSION}

Although further studies are needed to clarify the clinical value of candidate prognostic and predictive markers by immunohistochemical or genomic analysis, pathologists and clinicians should be prepared to consider such information beyond morphology for more personalised characterisation of prostate cancer. Given the new therapeutic opportunities, systematic testing for dMMR and HRD (at least BRCA1, $B R C A 2$, and $A T M$ ) should be offered to all patients with metastatic and/or castration resistant prostate cancer at this point in time. It is foreseeable that prostate-specific NGS panels for tissue and cfDNA analysis will play an increasing role in personalised molecular profiling and disease monitoring in the future.

Conflicts of interest and sources of funding: The authors state that there are no conflicts of interest to disclose.

Address for correspondence: Tatjana Vlajnic, MD, Institute of Pathology, University Hospital Basel, Schoenbeinstrasse 40, 4031, Basel, Switzerland. E-mail: tatjana.vlajnic@usb.ch

\section{References}

1. Gillessen S, Attard G, Beer TM, et al. Management of patients with advanced prostate cancer: report of the advanced prostate cancer consensus conference 2019. Eur Urol 2020; 77: 508-47.

2. Troyer DA, Jamaspishvili T, Wei W, et al. A multicenter study shows PTEN deletion is strongly associated with seminal vesicle involvement and extracapsular extension in localized prostate cancer. Prostate 2015 75: $1206-15$

3. Jamaspishvili T, Berman DM, Ross AE, et al. Clinical implications of PTEN loss in prostate cancer. Nat Rev Urol 2018; 15: 222-34.

4. Lotan TL, Wei W, Ludkovski O, et al. Analytic validation of a clinicalgrade PTEN immunohistochemistry assay in prostate cancer by comparison with PTEN FISH. Mod Pathol 2016; 29: 904-14.

5. Lotan TL, Gurel B, Sutcliffe S, et al. PTEN protein loss by immunostaining: analytic validation and prognostic indicator for a high risk surgical cohort of prostate cancer patients. Clin Cancer Res 2011; 17 $6563-73$.

6. Lotan TL, Wei W, Morais CL, et al. PTEN loss as determined by clinical-grade immunohistochemistry assay is associated with worse recurrence-free survival in prostate cancer. Eur Urol Focus 2016; 2 180-8.

7. Bismar TA, Yoshimoto M, Vollmer RT, et al. PTEN genomic deletion is an early event associated with ERG gene rearrangements in prostate cancer. BJU Int 2011; 107: 477-85.

8. Krohn A, Freudenthaler F, Harasimowicz S, et al. Heterogeneity and chronology of PTEN deletion and ERG fusion in prostate cancer. Mod Pathol 2014; 27: 1612-20.

9. Yoshimoto M, Joshua AM, Cunha IW, et al. Absence of TMPRSS2 ERG fusions and PTEN losses in prostate cancer is associated with a favorable outcome. Mod Pathol 2008; 21: 1451-60.

10. King JC, Xu J, Wongvipat J, et al. Cooperativity of TMPRSS2-ERG with PI3-kinase pathway activation in prostate oncogenesis. Nat Genet 2009; 41: 524-6.

11. Han B, Mehra R, Lonigro RJ, et al. Fluorescence in situ hybridization study shows association of PTEN deletion with ERG rearrangemen during prostate cancer progression. Mod Pathol 2009; 22: 1083-93.

12. Reid AH, Attard G, Ambroisine L, et al. Molecular characterisation of ERG, ETV1 and PTEN gene loci identifies patients at low and high risk of death from prostate cancer. Br J Cancer 2010; 102: 678-84.

13. Kumar-Sinha C, Tomlins SA, Chinnaiyan AM. Recurrent gene fusions in prostate cancer. Nat Rev Cancer 2008; 8: 497-511. 
14. Pettersson A, Graff RE, Bauer SR, et al. The TMPRSS2:ERG rear rangement, ERG expression, and prostate cancer outcomes: a cohor study and meta-analysis. Cancer Epidemiol Biomark Prev 2012; 21 : $1497-509$.

15. Bismar TA, Hegazy S, Feng Z, et al. Clinical utility of assessing PTEN and ERG protein expression in prostate cancer patients: a proposed method for risk stratification. J Cancer Res Clin Oncol 2018; 144: $2117-25$.

16. Ahearn TU, Pettersson A, Ebot EM, et al. A prospective investigation of PTEN loss and ERG expression in lethal prostate cancer. J Natl Cancer Inst 2016; 108: djv346.

17. de Bono JS, De Giorgi U, Rodrigues DN, et al. Randomized phase II study evaluating Akt blockade with ipatasertib, in combination with abiraterone, in patients with metastatic prostate cancer with and without PTEN loss. Clin Cancer Res 2019; 25: 928-36.

18. Inwald EC, Klinkhammer-Schalke M, Hofstadter F, et al. Ki-67 is prognostic parameter in breast cancer patients: results of a large population-based cohort of a cancer registry. Breast Cancer Res Treat 2013; 139: 539-52.

19. Johannessen AL, Torp SH. The clinical value of Ki-67/MIB-1 labeling index in human astrocytomas. Pathol Oncol Res 2006; 12: 143-7.

20. Kankuri M, Soderstrom KO, Pelliniemi TT, Vahlberg T, Pyrhonen S, Salminen E. The association of immunoreactive p53 and Ki-67 with Tstage, grade, occurrence of metastases and survival in renal cell carcinoma. Anticancer Res 2006; 26: 3825-33.

21. Munstedt K, von Georgi R, Franke FE. Correlation between MIB1determined tumor growth fraction and incidence of tumor recurrence in early ovarian carcinomas. Cancer Invest 2004; 22: 185-94.

22. Miller TP, Grogan TM, Dahlberg S, et al. Prognostic significance of the Ki-67-associated proliferative antigen in aggressive non-Hodgkin' lymphomas: a prospective Southwest Oncology Group trial. Blood 1994; 83: 1460-6.

23. Dziegiel P, Salwa-Zurawska W, Zurawski J, Wojnar A, Zabel M. Prognostic significance of augmented metallothionein (MT) expression correlated with Ki-67 antigen expression in selected soft tissue sarcomas. Histol Histopathol 2005; 20: 83-9.

24. Berlin A, Castro-Mesta JF, Rodriguez-Romo L, et al. Prognostic role of Ki-67 score in localized prostate cancer: a systematic review and metaanalysis. Urol Oncol 2017; 35: 499-506.

25. Lotan TL, Tomlins SA, Bismar TA, et al. Report from the International Society of Urological Pathology (ISUP) Consultation Conference on Molecular Pathology of Urogenital Cancers. Am J Surg Pathol 2020; 44: e15-29.

26. Petrelli F, Viale G, Cabiddu M, Barni S. Prognostic value of different cut-off levels of Ki-67 in breast cancer: a systematic review and metaanalysis of 64,196 patients. Breast Cancer Res Treat 2015; 153 477-91.

27. Maranta AF, Broder S, Fritzsche C, et al. Do you know the Ki-67 index of your breast cancer patients? Knowledge of your institution's Ki-67 index distribution and its robustness is essential for decision-making in early breast cancer. Breast 2020; 51: 120-6.

28. Senkus E, Kyriakides S, Ohno S, et al. Primary breast cancer: ESMO Clinical Practice Guidelines for diagnosis, treatment and follow-up. Ann Oncol 2015; 26 (Suppl 5): v8-30.

29. Duffy MJ, Harbeck N, Nap M, et al. Clinical use of biomarkers in breast cancer: updated guidelines from the European Group on Tumor Markers (EGTM). Eur J Cancer 2017; 75: 284-98.

30. Eggener SE, Rumble RB, Armstrong AJ, et al. Molecular biomarkers in localized prostate cancer: ASCO guideline. J Clin Oncol 2020; 38: 1474-94.

31. Salami SS, Hovelson DH, Kaplan JB, et al. Transcriptomic heterogeneity in multifocal prostate cancer. JCI Insight 2018; 3: e123468.

32. Galletti G, Leach BI, Lam L, Tagawa ST. Mechanisms of resistance to systemic therapy in metastatic castration-resistant prostate cancer Cancer Treat Rev 2017; 57: 16-27.

33. Antonarakis ES, Lu C, Wang $\mathrm{H}$, et al. AR-V7 and resistance to enzalutamide and abiraterone in prostate cancer. N Engl J Med 2014; 371 $1028-38$.

34. Bernemann C, Schnoeller TJ, Luedeke M, et al. Expression of AR-V7 in circulating tumour cells does not preclude response to next generation androgen deprivation therapy in patients with castration resistant prostate cancer. Eur Urol 2017; 71: 1-3.

35. Armstrong AJ, Halabi S, Luo J, et al. Prospective multicenter validation of androgen receptor splice variant 7 and hormone therapy resistance in high-risk castration-resistant prostate cancer: the PROPHECY study. J Clin Oncol 2019; 37: 1120-9.

36. Hench IB, Cathomas R, Costa L, et al. Analysis of AR/ARV7 expres sion in isolated circulating tumor cells of patients with metastatic castration-resistant prostate cancer (SAKK 08/14 IMPROVE Trial). Cancers 2019; 11: 1099.
37. Sharp A, Coleman I, Yuan W, et al. Androgen receptor splice variant-7 expression emerges with castration resistance in prostate cancer. J Clin Invest 2019; 129: 192-208.

38. Shaikhibrahim Z, Offermann A, Braun M, et al. MED12 overexpression is a frequent event in castration-resistant prostate cancer. Endocr Relat Cancer 2014; 21: 663-75.

39. Annala M, Vandekerkhove G, Khalaf D, et al. Circulating tumor DNA genomics correlate with resistance to abiraterone and enzalutamide in prostate cancer. Cancer Discov 2018; 8: 444-57.

40. Sumiyoshi T, Mizuno K, Yamasaki T, et al. Clinical utility of androgen receptor gene aberrations in circulating cell-free DNA as a biomarker for treatment of castration-resistant prostate cancer. Sci Rep 2019; 9: 4030.

41. Hovelson DH, Liu CJ, Wang Y, et al. Rapid, ultra low coverage copy number profiling of cell-free DNA as a precision oncology screening strategy. Oncotarget 2017; 8: 89848-66.

42. Aggarwal R, Huang J, Alumkal JJ, et al. Clinical and genomic characterization of treatment-emergent small-cell neuroendocrine prostate cancer: a multi-institutional prospective study. J Clin Oncol 2018; 36 2492-503.

43. Epstein JI, Amin MB, Beltran H, et al. Proposed morphologic classification of prostate cancer with neuroendocrine differentiation. Am J Surg Pathol 2014; 38: 756-67.

44. Kaur H, Samarska I, Lu J et al. Neuroendocrine differentiation in usualtype prostatic adenocarcinoma: molecular characterization and clinical significance. Prostate 2020; 80: 1012-23.

45. Bellur S, Van der Kwast T, Mete O. Evolving concepts in prostatic neuroendocrine manifestations: from focal divergent differentiation to amphicrine carcinoma. Hum Pathol 2019; 85: 313-27.

46. Jeetle SS, Fisher G, Yang ZH, et al. Neuroendocrine differentiation does not have independent prognostic value in conservatively treated prostate cancer. Virchows Arch 2012; 461: 103-7.

47. Beltran H, Prandi D, Mosquera JM, et al. Divergent clonal evolution of castration-resistant neuroendocrine prostate cancer. Nat Med 2016; 22: $298-305$.

48. Schelling LA, Williamson SR, Zhang S, et al. Frequent TMPRSS2-ERG rearrangement in prostatic small cell carcinoma detected by fluorescence in situ hybridization: the superiority of fluorescence in situ hybridization over ERG immunohistochemistry. Hum Pathol 2013; 44: 2227-33.

49. Hodgson DR, Dougherty BA, Lai Z, et al. Candidate biomarkers of PARP inhibitor sensitivity in ovarian cancer beyond the BRCA genes. Br J Cancer 2018; 119: 1401-9.

50. Pommier Y, O'Connor MJ, de Bono J. Laying a trap to kill cancer cells: PARP inhibitors and their mechanisms of action. Sci Transl Med 2016; 8: 362 ps 17.

51. Antonarakis ES. Abiraterone plus olaparib in prostate cancer: a new form of synthetic lethality? Lancet Oncol 2018; 19: 860-1.

52. Gadducci A, Guerrieri ME. PARP inhibitors alone and in combination with other biological agents in homologous recombination deficient epithelial ovarian cancer: from the basic research to the clinic. Crit Rev Oncol Hematol 2017; 114: 153-65.

53. Sunada S, Nakanishi A, Miki Y Crosstalk of DNA double-strand break repair pathways in poly(ADP-ribose) polymerase inhibitor treatment of breast cancer susceptibility gene 1/2-mutated cancer. Cancer Sci 2018 109: 893-9.

54. Bitler BG, Watson ZL, Wheeler LJ, Behbakht K. PARP inhibitors: clinical utility and possibilities of overcoming resistance. Gynecol Oncol 2017; 147: 695-704.

55. Robinson D, Van Allen EM, Wu YM, et al. Integrative clinical genomics of advanced prostate cancer. Cell 2015; 161: 1215-28.

56. Abida W, Armenia J, Gopalan A, et al. Prospective genomic profiling of prostate cancer across disease states reveals germline and somatic alterations that may affect clinical decision making. JCO Precis Oncol 2017; 2017: PO.17.00029.

57. Pritchard CC, Mateo J, Walsh MF, et al. Inherited DNA-repair gene mutations in men with metastatic prostate cancer. N Engl J Med 2016; 375: $443-53$.

58. Schweizer MT, Antonarakis ES, Bismar TA, et al. Genomic characterization of prostatic ductal adenocarcinoma identifies a high prevalence of DNA repair gene mutations. JCO Precis Oncol 2019; 3 : PO.18.00327.

59. Lotan TL, Kaur HB, Alharbi AM, Pritchard CC, Epstein JI. DNA damage repair alterations are frequent in prostatic adenocarcinomas with focal pleomorphic giant-cell features. Histopathology 2019; 74: 836-43.

60. Velho PI, Lim D, Wang H, et al. Molecular characterization and clinical outcomes of primary Gleason pattern 5 prostate cancer after radical prostatectomy. JCO Precis Oncol 2019; 3: PO.19.00081.

61. Risbridger GP, Taylor RA, Clouston D, et al. Patient-derived xenografts reveal that intraductal carcinoma of the prostate is a prominent pathol ogy in BRCA2 mutation carriers with prostate cancer and correlates with poor prognosis. Eur Urol 2015; 67: 496-503. 
62. Khani F, Wobker SE, Hicks JL, et al. Intraductal carcinoma of the prostate in the absence of high-grade invasive carcinoma represents a molecularly distinct type of in situ carcinoma enriched with oncogenic driver mutations. J Pathol 2019; 249: 79-89.

63. Mateo J, Carreira S, Sandhu S, et al. DNA-repair defects and olaparib in metastatic prostate cancer. N Engl J Med 2015; 373: 1697-708.

64. de Bono J, Mateo J, Fizazi K, et al. Olaparib for metastatic castrationresistant prostate cancer. N Engl J Med 2020; 382: 2091-102.

65. Abida W, Patnaik A, Campbell D, et al. Rucaparib in men with metastatic castration-resistant prostate cancer harboring a BRCA1 or BRCA2 gene alteration. J Clin Oncol 2020; Aug 14: JCO2001035.

66. Mateo J, Boysen G, Barbieri CE, et al. DNA repair in prostate cancer: biology and clinical implications. Eur Urol 2017; 71: 417-25.

67. Smith MR, Fizazi K, Sandhu SK, et al. Niraparib in patients (pts) with metastatic castration-resistant prostate cancer (mCRPC) and biallelic DNA-repair gene defects (DRD): correlative measures of tumor response in phase II GALAHAD study. J Clin Oncol 2020; 38 (6 Suppl): 118 .

68. Karzai F, Madan RA, Owens H, et al. A phase 2 study of olaparib and durvalumab in metastatic castrate-resistant prostate cancer (mCRPC) in an unselected population. J Clin Oncol 2018; 36 (6 Suppl): 163.

69. Pomerantz MM, Spisak S, Jia L, et al. The association between germline BRCA2 variants and sensitivity to platinum-based chemotherapy among men with metastatic prostate cancer. Cancer 2017; 123: 3532-9.

70. Cheng HH, Pritchard CC, Boyd T, Nelson PS, Montgomery B. Biallelic inactivation of BRCA2 in platinum-sensitive metastatic castrationresistant prostate cancer. Eur Urol 2016; 69: 992-5.

71. Kumar A, Coleman I, Morrissey C, et al. Substantial interindividual and limited intraindividual genomic diversity among tumors from men with metastatic prostate cancer. Nat Med 2016; 22: 369-78.

72. Mohler JL, Antonarakis ES, Armstrong AJ, et al. Prostate cancer, version 2.2019, NCCN clinical practice guidelines in oncology. J Natl Comp Cancer Netw 2019; 17: 479-505.

73. Antonarakis ES, Gomella LG, Petrylak DP. When and how to use PARP inhibitors in prostate cancer: a systematic review of the literature with an update on on-going trials. Eur Urol Oncol 2020; 3: 594-611.

74. Mateo J, Seed G, Bertan C et al Genomics of lethal prostate cancer at diagnosis and castration resistance. J Clin Invest 2020; 130: 1743-51.

75. Moreno JG, Gomella LG. Evolution of the liquid biopsy in metastatic prostate cancer. Urology 2019; 132: 1-9.

76. Imanishi S, Naoi Y, Shimazu K, et al. Clinicopathological analysis of homologous recombination-deficient breast cancers with special reference to response to neoadjuvant paclitaxel followed by FEC. Breast Cancer Res Treat 2019; 174: 627-37.

77. Wu YM, Cieslik M, Lonigro RJ. Vet al. Inactivation of CDK12 delineates a distinct immunogenic class of advanced prostate cancer. Cell 2018; 173: 1770-82e14

78. Pritchard CC, Morrissey C, Kumar A, et al. Complex MSH2 and MSH6 mutations in hypermutated microsatellite unstable advanced prostate cancer. Nat Commun 2014; 5: 4988.

79. Rodrigues DN, Rescigno P, Liu D, et al. Immunogenomic analyse associate immunological alterations with mismatch repair defects in prostate cancer. J Clin Invest 2018; 128: 5185.

80. Abida W, Cheng ML, Armenia J, et al. Analysis of the prevalence of microsatellite instability in prostate cancer and response to immune checkpoint blockade. JAMA Oncol 2019; 5: 471-8.

81. Le DT, Durham JN, Smith KN, et al. Mismatch repair deficiency predicts response of solid tumors to PD-1 blockade. Science 2017; 357: 409-13.
82. Isaacsson Velho P, Antonarakis ES. PD-1/PD-L1 pathway inhibitors in advanced prostate cancer. Expert Rev Clin Pharmacol 2018; 11 : $475-86$.

83. Antonarakis ES, Shaukat F, Isaacsson Velho P, et al. Clinical features and therapeutic outcomes in men with advanced prostate cancer and DNA mismatch repair gene mutations. Eur Urol 2019; 75: 378-82.

84. Albero-Gonzalez R, Hernandez-Llodra S, Juanpere N, et al. Immunohistochemical expression of mismatch repair proteins (MSH2, MSH6, MLH1, and PMS2) in prostate cancer: correlation with grade groups (WHO 2016) and ERG and PTEN status. Virchows Arch 2019; 475: $223-31$.

85. Evrard C, Tachon G, Randrian V, Karayan-Tapon L, Tougeron D. Microsatellite instability: diagnosis, heterogeneity, discordance, and clinical impact in colorectal cancer. Cancers 2019; 11: 1567.

86. Perner S, Hofer MD, Kim R, et al. Prostate-specific membrane antigen expression as a predictor of prostate cancer progression. Hum Pathol 2007; 38: 696-701.

87. Joung JY, Cho KS, Chung HS, et al. Prostate specific membrane antigen mRNA in blood as a potential predictor of biochemical recurrence after radical prostatectomy. J Korean Med Sci 2010; 25: 1291-5.

88. Hofman MS, Violet J, Hicks RJ, et al. [(177)Lu]-PSMA-617 radionuclide treatment in patients with metastatic castration-resistant prostate cancer (LuPSMA trial): a single-centre, single-arm, phase 2 study. Lancet Oncol 2018; 19: 825-33.

89. Prive BM, Janssen MJR, van Oort IM, et al. Lutetium-177-PSMA-I\&T as metastases directed therapy in oligometastatic hormone sensitive prostate cancer, a randomized controlled trial. BMC Cancer 2020; 20: 884.

90. Ferraro DA, Ruschoff JH, Muehlematter UJ, et al. Immunohistochemical PSMA expression patterns of primary prostate cancer tissue are associated with the detection rate of biochemical recurrence with (68) Ga-PSMA-11-PET. Theranostics 2020; 10: 6082-94.

91. Woythal N, Arsenic R, Kempkensteffen C, et al. Immunohistochemica validation of PSMA expression measured by (68)Ga-PSMA PET/CT in primary prostate cancer. J Nucl Med 2018; 59: 238-43.

92. Gasi Tandefelt D, de Bono J. Circulating cell-free DNA: translating prostate cancer genomics into clinical care. Mol Aspects Med 2020; 72: 100837.

93. Vidula N, Rich TA, Sartor O, et al. Routine plasma-based genotyping to comprehensively detect germline, somatic, and reversion BRCA mutations among patients with advanced solid tumors. Clin Cancer Res 2020; 26: $2546-55$.

94. Wyatt AW, Annala M, Aggarwal R, et al. Concordance of circulating tumor DNA and matched metastatic tissue biopsy in prostate cancer. J Natl Cancer Inst 2017; 109: djx118.

95. Filges S, Yamada E, Stahlberg A, Godfrey TE. Impact of polymerase fidelity on background error rates in next-generation sequencing with unique molecular identifiers/barcodes. Sci Rep 2019; 9: 3503.

96. Clark TA, Chung JH, Kennedy M, et al. Analytical validation of a hybrid capture-based next-generation sequencing clinical assay for genomic profiling of cell-free circulating tumor DNA. J Mol Diagn 2018; 20: 686-702.

97. Ledet EM, Lilly MB, Sonpavde G, et al. Comprehensive analysis of AR alterations in circulating tumor DNA from patients with advanced prostate cancer. Oncologist 2020; 25: 327-33.

98. Taavitsainen S, Annala M, Ledet E, et al. Evaluation of commercia circulating tumor DNA test in metastatic prostate cancer. JCO Precis Oncol 2019; 3: PO.19.00014. 